\title{
The accretion/diffusion theory for $\lambda$ Bootis stars in the light of spectroscopic data
}

\author{
U. Heiter ${ }^{1,2}$, W. W. Weiss ${ }^{1}$, and E. Paunzen ${ }^{1,3}$ \\ 1 Institute for Astronomy (IfA), University of Vienna, Türkenschanzstrasse 17, 1180 Vienna, Austria \\ e-mail: weiss@astro.univie.ac.at \\ 2 Department of Astronomy, Case Western Reserve University, 10900 Euclid Avenue, Cleveland, OH 44106-7215, \\ USA \\ 3 Zentraler Informatikdienst der Universität Wien, Universitätsstrasse 7, 1010 Vienna, Austria \\ e-mail: ernst.paunzen@univie.ac.at
}

Received 18 May 2001 / Accepted 25 October 2001

\begin{abstract}
Most of the current theories suggest the $\lambda$ Bootis phenomenon to originate from an interaction between the stellar surface and its local environment. In this paper, we compare the abundance pattern of the $\lambda$ Bootis stars to that of the interstellar medium and find larger deficiencies for $\mathrm{Mg}, \mathrm{Si}, \mathrm{Mn}$ and $\mathrm{Zn}$ than in the interstellar medium. A comparison with metal poor post-AGB stars showing evidence for circumstellar material indicates a similar physical process possibly being at work for some of the $\lambda$ Bootis stars, but not for all of them. Despite the fact that the number of spectroscopically analysed $\lambda$ Bootis stars has considerably increased in the past, a test of predicted effects with observations shows current abundance and temperature data to be still controversial.
\end{abstract}

Key words. stars: abundances - stars: atmospheres - stars: chemically peculiar - stars: early-type ISM: abundances

\section{Introduction}

In the last few years, the $\lambda$ Bootis stars (metal-poor population I A to F type stars) have experienced increased attention by abundance analysis groups. The results have been collected by Heiter (2002, hereafter referred to as Paper I) and show that the proportion of $\lambda$ Bootis stars with known abundances is now large enough to examine the abundances with respect to other stellar parameters on a good statistical basis. The analysed stars span a wide range of atmospheric parameters, in particular the effective temperature (Fig. 1). This parameter plays a major role in current theories, which are briefly reviewed in the following.

Venn \& Lambert (1990) proposed that the peculiar abundance patterns of $\lambda$ Bootis stars originate from the interstellar medium, which shows a similar abundance distribution (see Sect. 3). Within this hypothesis it is assumed that only the interstellar gas, but not the dust, is accreted onto the surface of the stars. Charbonneau (1991) calculated the concentration of the elements Ca, $\mathrm{Ti}, \mathrm{Mn}$ and $\mathrm{Eu}$ in the superficial convection zone (SCZ) within a simple analytical model, which takes into account

Send offprint requests to: U. Heiter, e-mail: ulrike@fafnir.astr.cwru.edu accretion of interstellar gas and diffusion below the SCZ, for various effective temperatures. It is assumed that the atmospheric material is mixed thoroughly from the surface to the bottom of the SCZ.

More detailed numerical calculations have been performed by Turcotte \& Charbonneau (1993) for the elements Ca, Sc and Ti. Abundance profiles of the first two elements show overabundances at the surface if only chemical separation and convective mixing is taken into account, whereas $\mathrm{Ti}$ is predicted to be underabundant in this case. If accretion of circumstellar gas with a certain amount of depletion is added, the calculations show that for an accretion rate $\dot{M}$ of at least $5 \times 10^{-14} M_{\odot} \mathrm{yr}^{-1}$, the abundances of the examined elements in the convection zone converge to the values in the accreted gas on a very short timescale. Two other points became evident. Independently of $\dot{M}$ and the duration of the accretion phase, the abundances are again governed by chemical separation when accretion is stopped. This means that accretion must be an ongoing process if it is responsible for the observed abundance pattern. Secondly, meridional circulation induced by rotation with equatorial velocities up to $125 \mathrm{~km} \mathrm{~s}^{-1}$ does not alter the surface abundances produced by accretion. Higher rotation rates could not be treated due to numerical problems. All these calculations 


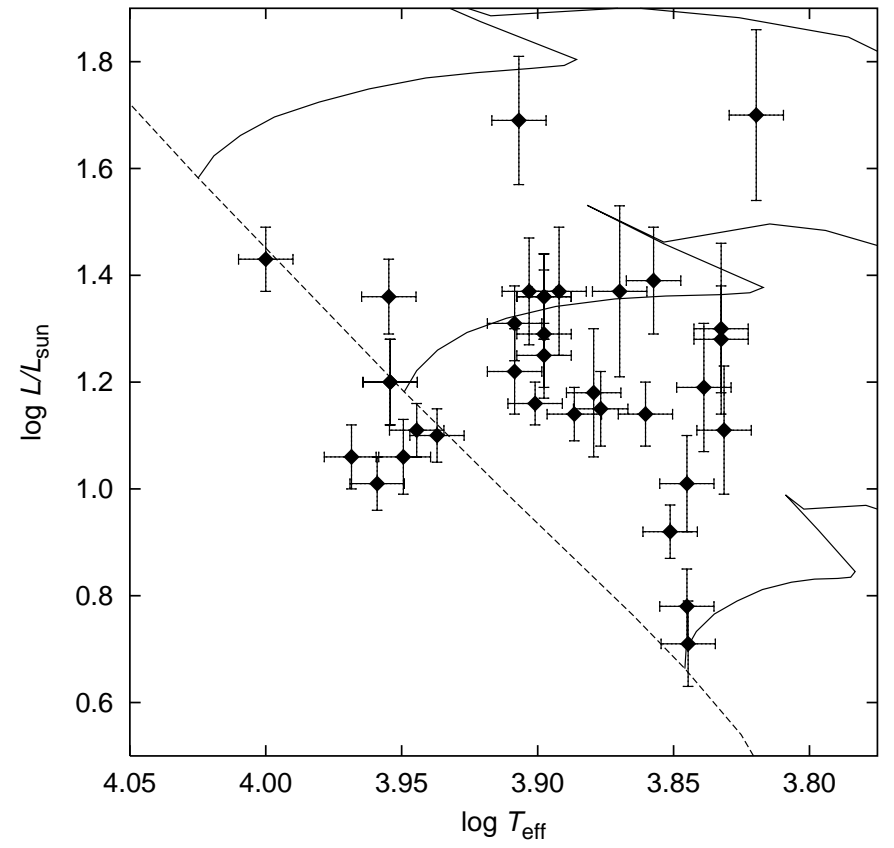

Fig. 1. Theoretical HR diagram for all $\lambda$ Bootis stars with known abundances. The luminosities have been derived from Hipparcos measurements, if available, and from Strömgren photometry otherwise, as described in Paunzen (2000), using data from Hauck \& Mermilliod (1990), Handler (1995) and Martinez et al. (1998). Evolutionary tracks for $1.5 M_{\odot}, 2 M_{\odot}$ and 2.5 $M_{\odot}$ taken from Claret (1995) are also shown.

are based on only one static stellar envelope model with a fixed parameter set of $\left(T_{\text {eff }}, \log g\right)=(8000 \mathrm{~K}, 4.3)$.

The separation of gas and dust in a circumstellar shell was investigated by Andrievsky \& Paunzen (2000), who calculated gas and dust grain velocities in a shell extending to 100 stellar radii around a star with $T_{\text {eff }}=8500 \mathrm{~K}$, assuming a polytropic density distribution. For a ratio between radiative and gravitational acceleration on the gas of 0.99 , large dust grains and a rather smooth density distribution (polytropic index $=2$ ), they indeed find dust grains to be forced to an outward motion by radiative pressure. The separation becomes effective at a distance from the stellar surface where the temperature is about $1600 \mathrm{~K}$ (condensation temperature for heavy elements), which corresponds to about 10 stellar radii. The gaseous part of the shell is accreted to the surface of the star. Thus the two components are decoupled and the superficial chemical composition is changed according to the depletions in the gas coming from the outer part of the shell. The calculations take into account only interactions between neutral particles because they are shown to be more important than Coulomb-type interactions. Within this simple model only rough estimates for the gas-dust separation can be made, which are based on very restricted assumptions. But it could serve for more sophisticated models, which in particular should be extended to lower temperatures and smaller dust grains, which are more likely to be formed around $\lambda$ Bootis stars.
An earlier theoretical approach to the $\lambda$ Bootis phenomenon had its origin in recalling the physical processes operating in the atmospheres of Am stars. The Am abundance pattern has been explained by Charbonneau \& Michaud (1991) by chemical separation of elements below the superficial hydrogen convection zone, caused by diffusion processes. In order to produce the actual abundance values of Am stars, an additional process is needed, e.g. a small amount of mass-loss $\left(10^{-15} M_{\odot} \mathrm{yr}^{-1}\right)$. Evidence for mass-loss has not yet been observed for Am stars. By introducing a two orders of magnitude higher mass-loss rate, Michaud \& Charland (1986) have changed the calculated Am abundance pattern to a $\lambda$ Bootis like one, but they have not been able to produce underabundances as low as were observed in several $\lambda$ Bootis stars. The underabundances even vanish for most elements if meridional circulation induced by high rotational velocities is taken into account (Charbonneau 1993). Although large uncertainties are still involved in the modeling (above all for the radiative acceleration), this theory has been widely discarded as an explanation of the $\lambda$ Bootis star abundances.

Further theoretical considerations include Andrievsky (1997), who proposed that $\lambda$ Bootis stars are the result of a merger of contact binaries of W UMa type. He argues that mass loss during the merger phase could form the circumstellar shell, whose accretion leads to the observed underabundances. The hypothesis is substantiated by lifetime and number estimates. Faraggiana \& Bonifacio (1999) suspect that a part of the $\lambda$ Bootis stars are undetected spectroscopic binary systems, and that their abundance anomalies are due to veiling effects in the composite spectra.

Summarizing, in the mentioned theories the $\lambda$ Bootis phenomenon seems to originate from an interaction between the stellar surface and its local environment. In the following we confront predicted effects with observations.

\section{Examination of stellar parameters and abundances}

\subsection{Correlations between stellar parameters}

First, we searched for correlations among the atmospheric parameters $T_{\text {eff }}$ and $\log g$, the projected rotational velocity, the relative age $\left(t_{\text {rel }}\right)$ and the pulsational period, all listed in Table 1. A correlation appeared to exist between $T_{\text {eff }}$ and $\log g$ and also between $t_{\text {rel }}-T_{\text {eff }}$ and $t_{\text {rel }}-\log g$. In all three cases the errors of the slopes were smaller than $20 \%$. However, these correlations seem to be due to a selection effect, which becomes clear from Fig. 1, showing the location of the analysed stars in the HR diagram, as well as some evolutionary tracks from Claret (1995). The lack of young stars in cooler regions and old stars in hotter regions is evident. This imbalance disappears when the same diagram is regarded for all $\lambda$ Bootis stars (Paunzen 2000, Fig. 33).

The $T_{\text {eff }}-v \cdot \sin i$ relationship basically follows that of normal dwarfs (Schmidt-Kaler 1982), which shows that 
Table 1. Stellar parameters of the $\lambda$ Bootis stars for which abundance analyses have been performed up to now. The atmospheric parameters have been taken from the sources listed in Table 6 of Paper I. The column labeled $\sigma[\mathrm{X}]$ lists the standard deviations of 7-9 heavy element abundances as described in the text. The age $t_{\text {rel }}$ is given relative to the main sequence lifetime and has been estimated, like the mass, using the CESAM code (Morel 1997). The pulsational parameters are from Paunzen et al. (1997, 1998). Column $A / U L$ gives amplitudes for variable and upper limits for constant stars with the used filter in parentheses. The coordinates have been extracted from the Simbad database. The distances $r$ have been calculated using Hipparcos parallaxes (Perryman \& ESA 1997). The errors for $v \cdot \sin i$ and $r$ are given in parentheses.

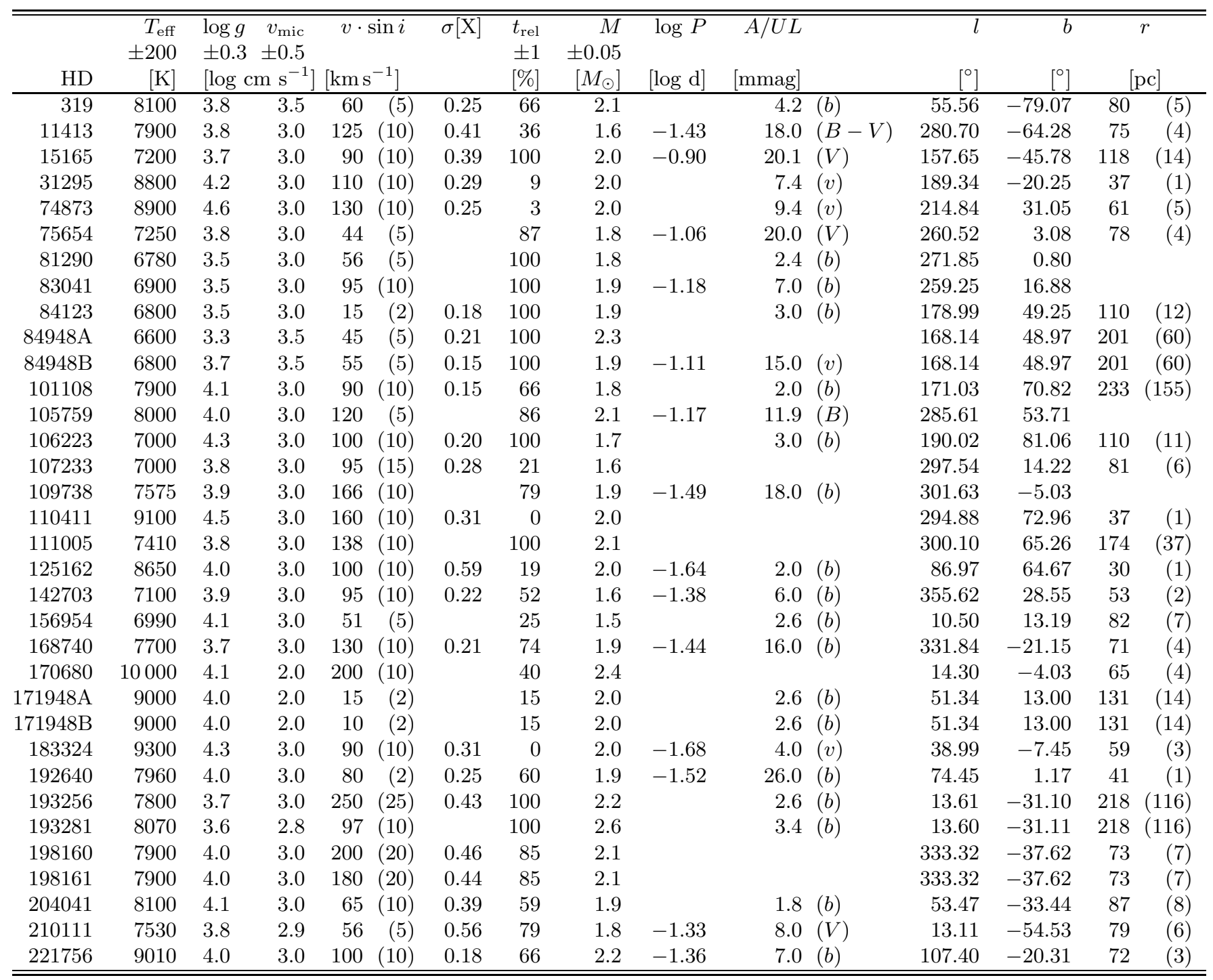

a high $v \cdot \sin i$ value cannot be used as a $\lambda$ Bootis classification criterion. No correlation exists between $v \cdot \sin i$ and $t_{\text {rel }}$.

\subsection{Correlations between stellar parameters and abundances}

As a next step, a linear regression, weighted by $1 / s^{2}$ according to the errors of the abundances $(s$, taken from the references given in Table 6 of Paper I), has been computed for each element with respect to each of the five parameters discussed in the previous subsection. For most elements the errors of the slopes appeared to be very large compared to their values. For some elements with an error between 55 and $100 \%$ a very weak correlation might be indicated. These cases are marked in Table 2 with one plus sign (positive slope) or one minus sign (negative slope). For $\mathrm{Cr}, \mathrm{Fe}$ and $\mathrm{Sr}$ vs. $T_{\text {eff }}$, for Sc vs. $t_{\text {rel }}$, for Ca vs. $\log P$ and for Mg vs. $v \cdot \sin i$, the errors of the slopes amount to about $50 \%$, and they lie between 30 and $40 \%$ for Sc vs. $T_{\text {eff }}, \mathrm{C}$ and $\mathrm{O}$ vs. $\log g, \mathrm{Na}$ and $\mathrm{Sr}$ vs. $t_{\text {rel }}, \mathrm{Mg}$ vs. $\log P$ and $\mathrm{Si}$ and $\mathrm{Fe}$ vs. $v \cdot \sin i$. These cases may indicate a weak correlation and are represented by two plus or minus signs in Table 2. Some of them are shown in Fig. 2, together with $95 \%$ confidence bands of the fits. However, within a $90 \%$ confidence level the hypothesis of no correlation between parameters and abundances is possible for all elements and parameters. Plots for the same elements 

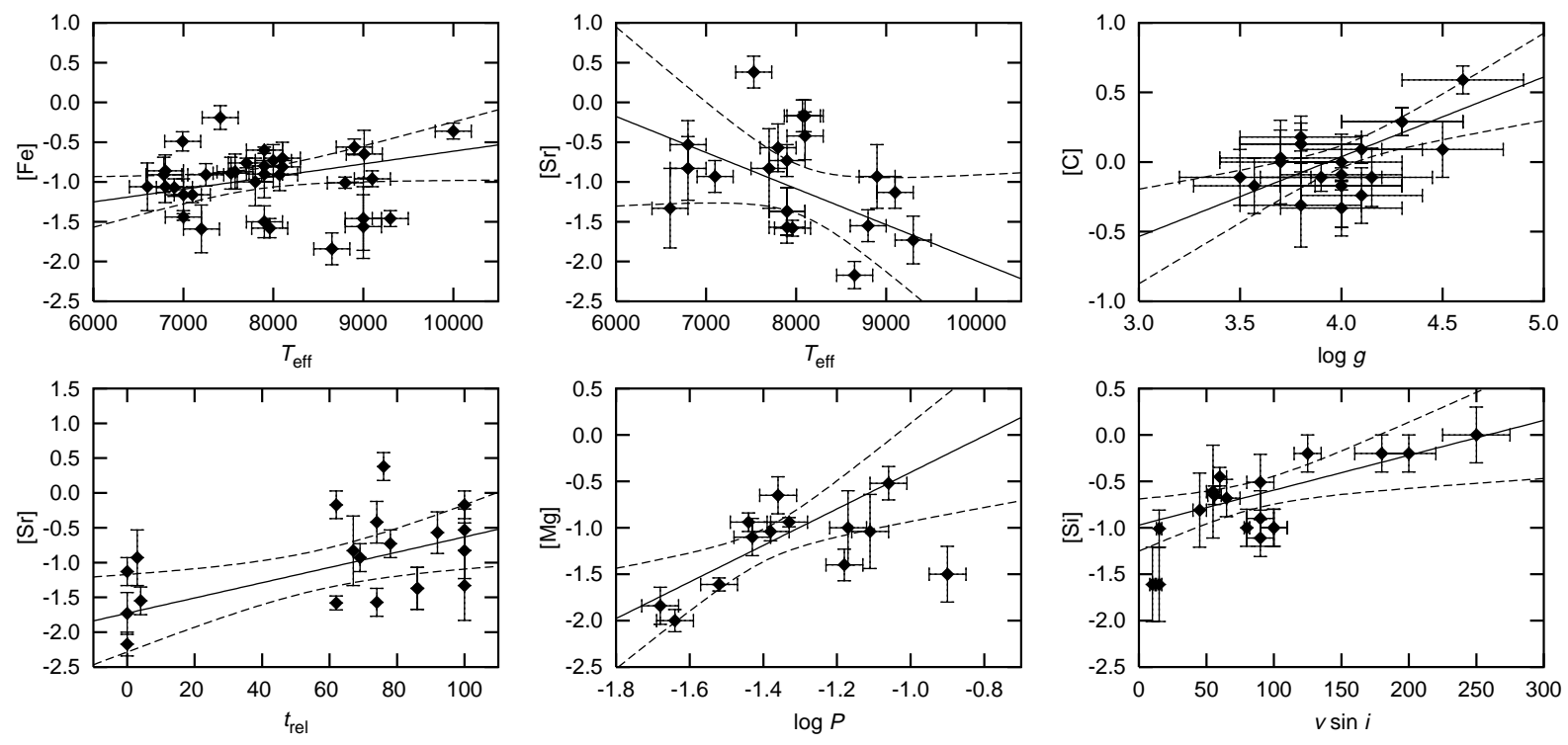

Fig. 2. Dependences of element abundances on the different parameters. This figure contains only "best cases", to avoid overloading this paper with graphs. The dashed lines indicate the $95 \%$ confidence bands of the weighted linear fit (solid line).

Table 2. Formal correlations between stellar parameters and abundances. Double signs indicate a weak correlation (error of the slope lower than 55\%), single signs indicate a very weak correlation (error of the slope between 55 and 100\%), whereas all other quantities are clearly uncorrelated. + and - signs correspond to positive and negative slopes.

\begin{tabular}{lrrrrr}
\hline \hline El. & $T_{\text {eff }}$ & $\log g$ & $t_{\text {rel }}$ & $\log P$ & $v \cdot \sin i$ \\
\hline $\mathrm{C}$ & & ++ & - & & + \\
$\mathrm{C}_{\mathrm{NIR}}$ & + & + & & & - \\
$\mathrm{O}$ & & -- & & & \\
$\mathrm{O}_{\mathrm{NIR}}$ & + & & - & & + \\
$\mathrm{Na}$ & & - & ++ & & + \\
$\mathrm{Mg}$ & & - & + & ++ & ++ \\
$\mathrm{Si}$ & - & & + & & ++ \\
$\mathrm{Ca}$ & & & + & ++ & \\
$\mathrm{Sc}$ & ++ & & -- & - & + \\
$\mathrm{Ti}$ & + & & + & + & + \\
$\mathrm{Cr}$ & ++ & & & & + \\
$\mathrm{Fe}$ & ++ & + & & + & + \\
$\mathrm{Ni}$ & + & + & & & - \\
$\mathrm{Sr}$ & -- & - & ++ & & - \\
$\mathrm{Ba}$ & & & & & \\
\hline \hline
\end{tabular}

and parameters for normal stars, where available (Fig. 3, see Paper I for the references), do not show any correlation except maybe for $[\mathrm{Si}]-v \cdot \sin i$.

\subsection{The effective temperature}

Within the accretion/diffusion model, the abundances depend mainly on the $\dot{M}$, the mass of the convection zone, and the radiative acceleration. One prediction of this model is that underabundances are most likely to appear in a certain temperature interval, in which accretion dominates the diffusion processes. This interval begins at $7000 \mathrm{~K}$ for all elements, and its width depends on the element and $\dot{M}$. For Ti, it extends at least to $9500 \mathrm{~K}$, for $\mathrm{Mn}$ at least to $8000 \mathrm{~K}$, and for Ca well over $12000 \mathrm{~K}$. For higher temperatures, the abundance effects depend more on $\dot{M}$ than for lower temperatures. Observational implications are (Charbonneau 1991):

Cool border: $7000 \mathrm{~K}$ for all accretion rates and elements. Hot border: abundance variations from element to element and from star to star should be more pronounced among the hotter $\lambda$ Bootis stars than among the cooler ones.

The most recent list of classified $\lambda$ Bootis stars can be found in Paunzen (2000). The temperatures of the stars subject to abundance analyses (Table 1) span the whole temperature range given by the stars in this list. In the temperature range of 6500 to $8500 \mathrm{~K}, 45 \%$ of the $56 \lambda$ Bootis stars, and in the range of 8500 to $10500 \mathrm{~K}$, about $55 \%$ of the $16 \lambda$ Bootis stars have been analysed. The theoretically predicted cool border of the $\lambda$ Bootis group is not supported by our observations because the coolest $\lambda$ Bootis star in our sample has a $T_{\text {eff }}$ of $6600 \mathrm{~K}$. However, the treatment of convection used in the accretion/diffusion model plays a crucial role for modeling these borders. For example, increasing $\alpha$ (the ratio of mixing length to pressure scale height) from 1.4 to 2.0 would shift the cool border for $\mathrm{Ti}$ by $400 \mathrm{~K}$ towards higher values (Charbonneau 1991). Similarly, a decrease of $\alpha$ (values of 1.25 down to 0.5 are commonly used in models of main sequence stars) or a different convection model could result in a shift towards lower temperatures.

For hot stars, the accretion/diffusion theory predicts different degrees of heavy element depletion. For an accretion rate of $5 \times 10^{-14} M_{\odot} \mathrm{yr}^{-1}$, Ca should still be underabundant for a $\lambda$ Bootis star above $9000 \mathrm{~K}$, while Ti can be solar abundant. This difference vanishes for $\dot{M} \geq 2 \times 10^{-13} M_{\odot} \mathrm{yr}^{-1}$ (Charbonneau 1991, Fig. 1). In all five stars with $T_{\text {eff }}$ greater than about $9000 \mathrm{~K}$ and abundances determined for Ca and Ti (this includes HD 31295), 

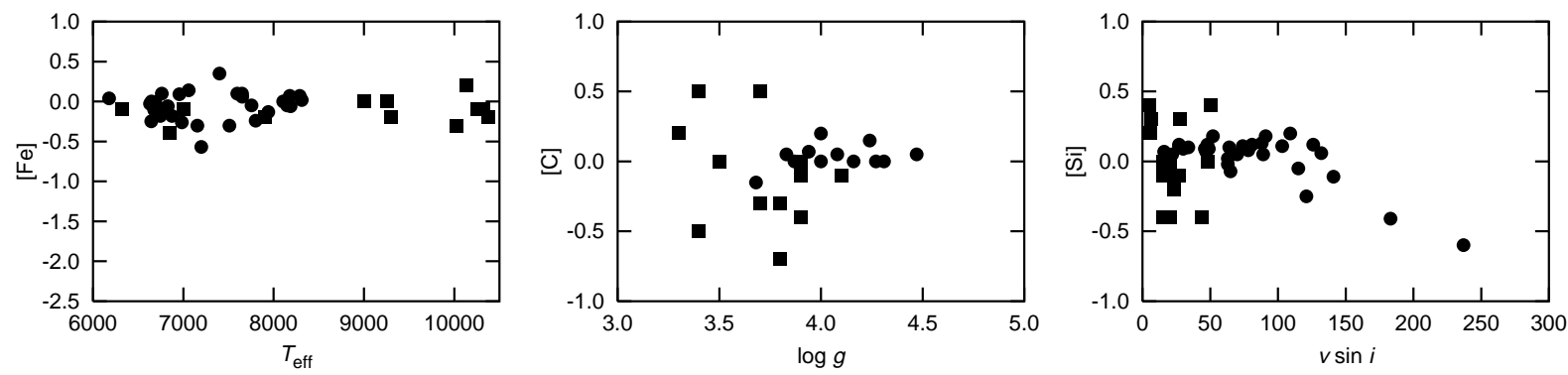

Fig. 3. Distribution of Fe, $\mathrm{C}$ and $\mathrm{Si}$ abundances with $T_{\mathrm{eff}}, \log g$ and $v \cdot \sin i$, respectively, for normal $\mathrm{A}$ and $\mathrm{F}$ stars in the Hyades (circles) and in the field (squares).

these elements have the same abundance. At the cool border, regardless of $\dot{M}$, all elements should become solar abundant at the same temperature. To test these predictions (arbitrarily extended to all elements) we calculated the standard deviations of the heavy element abundances (relative to solar) for individual stars, where at least seven of the elements $\mathrm{Mg}, \mathrm{Si}, \mathrm{Ca}, \mathrm{Sc}, \mathrm{Ti}, \mathrm{Cr}, \mathrm{Fe}, \mathrm{Sr}$ and Ba have been measured (see the column labelled $\sigma[\mathrm{X}]$ in Table 1). We did not find any dependence of the abundance scatter on effective temperature.

A positive trend of abundances with temperature is indicated for $\mathrm{Sc}, \mathrm{Cr}$ and $\mathrm{Fe}$ (Table 2). In the context of Charbonneau's model, this would imply a decrease of $\dot{M}$ with increasing $T_{\text {eff }}$. On the other hand, Sr shows a negative trend and $\mathrm{Na}$ behaves totally differently, because pronounced overabundances occur around $8000 \mathrm{~K}$ and they decrease towards both temperature borders.

For underabundances, the accretion/diffusion theory predicts a rather narrow temperature interval of 7000-9000 K for Mn and 7000-8000 K for Eu. The mean Mn abundance of the five $\lambda$ Bootis stars with temperatures from 6800 to $7250 \mathrm{~K}$ is $-1.2 \pm 0.3 \mathrm{dex}$, whereas the abundances of the remaining three stars $(\approx 8000 \mathrm{~K})$ span a large range $(-0.4,-1.0$ and $-2.1 \mathrm{dex})$, which might be caused by different accretion rates. Due to the lack of Eu abundance measurements, the behavior of this element cannot be tested. No accretion/diffusion calculations are available for the light elements, whose nearly solar abundances are characteristic for $\lambda$ Bootis stars.

The discussion above shows that current abundance and temperature data are inconclusive with regard to predictions of the accretion/diffusion theory.

\subsection{The other parameters}

The marginal C abundance correlation with $\log g$ (Fig. 2) could indicate a decrease with progressing evolution. On the other hand, Table 2 indicates an opposite trend for other elements. A connection to the stellar age is also present for the pulsational period via the $Q$-constant, which is the suspected reason for the positive slope present for the abundances of $\mathrm{Mg}, \mathrm{Ca}, \mathrm{Ti}$ and Fe with respect to $\log P$.
Charbonneau (1991) has calculated the critical values for the equatorial rotational velocity, for various effective temperatures and accretion rates, above which the rotational mixing is too strong for a star to show abundance anomalies (see his Fig. 2). These can be interpreted as upper limits for the $v \cdot \sin i$ values of $\lambda$ Bootis stars. For $\mathrm{Ti}$, the maximum velocities at $9000 \mathrm{~K}$ are $170 \mathrm{kms}^{-1}$ $\left(5 \times 10^{-14} M_{\odot} \mathrm{yr}^{-1}\right), 250 \mathrm{~km} \mathrm{~s}^{-1}\left(10^{-13} M_{\odot} \mathrm{yr}^{-1}\right)$ and $370 \mathrm{~km} \mathrm{~s}^{-1}\left(2 \times 10^{-13} M_{\odot} \mathrm{yr}^{-1}\right)$. The values of $v \cdot \sin i$ and $T_{\text {eff }}$ of the $\lambda$ Bootis stars in our sample are consistent with $\dot{M}=10^{-13} M_{\odot} \mathrm{yr}^{-1}$. The abundances of the heavy elements $\mathrm{Mg}, \mathrm{Si}$, Ti and Fe seem to increase with $v \cdot \sin i$ (Fig. 2 for the best case of Si and Table 2), which could indicate that rotational mixing prevents the development of large underabundances for higher rotational velocities. Note that an opposite trend seems to exist for normal stars (Fig. 3).

\section{Comparison with the interstellar medium}

The accretion hypothesis was proposed by Venn \& Lambert (1990) because they noticed similarities between $\lambda$ Bootis star underabundances and element depletions in the interstellar gas. They assumed that gas in a dusty circumstellar nebula, whose accretion could result in the observed underabundances, shows depletions similar to those of the interstellar gas. Therefore we compare in this section the abundance pattern of the $\lambda$ Bootis stars (Paper I) with recent determinations of abundances in the interstellar gas.

The chemical composition of the interstellar gas has been studied by several authors along many different sight lines. Savage \& Sembach (1996a) give an extensive review on abundances in the interstellar medium (ISM). The galactic longitudes, latitudes and distances of the stars at the end of the sight lines are given in columns two to four of Table 3. The hydrogen column density is given in column five and the depletions of six elements are given in columns six to eleven. The solar values have been taken from Grevesse et al. (1996). Abundances for the following additional elements, which have also been studied in $\lambda$ Bootis stars, are available: C, N, Na (Welty et al. 1994), $\mathrm{Al}, \mathrm{Ca}, \mathrm{Ti}, \mathrm{Cr}, \mathrm{Mn}, \mathrm{Co}, \mathrm{Ni}, \mathrm{Cu}$. For Na, only absorption lines of the neutral element have been studied, whereas 
Table 3. Characteristic parameters for the interstellar medium along the sight lines to several stars. The fifth column contains the total hydrogen column density, and the consecutive columns give for each element $X$ the value $\log \left(\frac{N(X)}{N(\mathrm{H})}\right)-\log \left(\frac{N(X)}{N(\mathrm{H})}\right)$. The unit of $N(X)$ is $\mathrm{cm}^{-2}$. Column twelve gives the references. The asterisks denote sight lines with coordinates in the vicinity of $\lambda$ Bootis stars. Errors are given in parentheses.

\begin{tabular}{|c|c|c|c|c|c|c|c|c|c|c|c|c|c|}
\hline$(1)$ & & $(2)$ & $(3)$ & \multicolumn{2}{|c|}{ (4) } & (5) & (6) & (7) & (8) & (9) & $(10)$ & (11) & (12) \\
\hline HD & & $l\left[^{\circ}\right]$ & $b\left[^{\circ}\right]$ & $r[]$ & c] & $\log N(\mathrm{H})$ & {$[\mathrm{O}]$} & {$[\mathrm{Mg}]$} & {$[\mathrm{Si}]$} & {$[\mathrm{S}]$} & {$[\mathrm{Fe}]$} & {$[\mathrm{Zn}]$} & Ref. \\
\hline 5394 & & 123.6 & -2.2 & 188 & $(22)$ & 20.18 & $-0.3(1)$ & & & & & & 1 \\
\hline 18100 & & 217.9 & -62.7 & 3100 & & 20.14 & & $-0.8(1)$ & $-0.4(1)$ & $-0.3(1)$ & $-0.8(1)$ & $-0.2(2)$ & 2,3 \\
\hline 22586 & $*$ & 264.2 & -50.4 & 2000 & & 20.35 & & & $-0.6(2)$ & $-0.6(2)$ & $-1.2(2)$ & & 2 \\
\hline 24398 & & 162.3 & -16.7 & 301 & $(88)$ & 21.20 & $-0.4(1)$ & & & & & & 1 \\
\hline 24760 & & 157.4 & -10.1 & 165 & $(26)$ & 20.52 & $-0.4(1)$ & & & & & & 1 \\
\hline 24912 & & 160.4 & -13.1 & 543 & (334) & 21.29 & $-0.4(1)$ & $-1.2(1)$ & & & $-1.9(1)$ & $-0.6(1)$ & $1,2,4$ \\
\hline 35149 & $*$ & 199.2 & -17.9 & 295 & (102) & 20.74 & & & & & & $-0.3(3)$ & 2,4 \\
\hline 36486 & $*$ & 203.9 & -17.7 & 281 & $(85)$ & 20.17 & $-0.4(1)$ & & & & & $-0.2(3)$ & 1,4 \\
\hline 36861 & $*$ & 195.1 & -12.0 & 324 & (109) & 20.81 & $-0.4(1)$ & & & & & & 1 \\
\hline 37043 & & 209.5 & -19.6 & 407 & (185) & 20.18 & $-0.3(1)$ & & & & & & 1 \\
\hline 37128 & $*$ & 205.2 & -17.2 & 412 & (246) & 20.48 & $-0.4(1)$ & & & & & $-0.2(3)$ & 1,4 \\
\hline 38666 & & 237.3 & -27.1 & 397 & (111) & 19.85 & & $-0.4(4)$ & $-0.5(3)$ & $0.0(1)$ & $-1.1(1)$ & $-0.1(1)$ & 2 \\
\hline 38771 & & 214.5 & -18.5 & 221 & $(45)$ & 20.53 & $-0.4(1)$ & & & & & & 1 \\
\hline 44743 & & 226.0 & -14.0 & 153 & (17) & 19.28 & & & $0.0(4)$ & & & & 5 \\
\hline 47839 & & 202.9 & 2.2 & 313 & (93) & 20.40 & $-0.5(1)$ & & & & & $-0.2(3)$ & 1,4 \\
\hline 57061 & & 238.2 & -5.5 & 1514 & & 20.80 & $-0.3(1)$ & & & & & $-0.2(3)$ & 1,4 \\
\hline 68273 & & 262.8 & -7.7 & 258 & (41) & 19.74 & & $-0.4(2)$ & $-0.7(2)$ & & $-1.1(3)$ & & 2 \\
\hline 72089 & & 263.2 & -3.9 & 1700 & & $20.60^{\dagger}$ & & $-1.1(2)$ & $-0.5(3)$ & & $-0.5(4)$ & & 2 \\
\hline 91316 & & 234.9 & 52.8 & 869 & & 20.44 & & & & & & $-0.2(3)$ & 4 \\
\hline 93521 & $*$ & 183.1 & 62.2 & 1700 & & 20.10 & & $0.0(4)$ & $-0.4(2)$ & $-0.1(1)$ & $-0.7(4)$ & & 2 \\
\hline 100340 & & 258.8 & 61.2 & 5300 & & 20.47 & & $-0.8(2)$ & & $-0.4(1)$ & & & 3 \\
\hline 116852 & & 304.9 & -16.1 & 4800 & & 20.96 & & $-0.8(2)$ & & $-0.3(2)$ & $-0.8(2)$ & $-0.3(2)$ & 2 \\
\hline 120086 & & 329.6 & 57.5 & 299 & (140) & 20.41 & & $-0.9(2)$ & $-0.7(2)$ & $-0.7(2)$ & $-1.3(2)$ & & 2 \\
\hline 141637 & $*$ & 346.1 & 21.7 & 160 & $(27)$ & 21.20 & & & & & & $-0.4(3)$ & 2,4 \\
\hline 143018 & $*$ & 347.2 & 20.2 & 141 & (19) & 20.75 & & & & & & $-0.3(3)$ & 2,4 \\
\hline 144217 & $*$ & 353.2 & 23.6 & 163 & (36) & 21.08 & & & & & & $-0.2(3)$ & 4 \\
\hline 147165 & $*$ & 351.3 & 17.0 & 225 & (50) & 21.40 & & & & & & $-0.4(3)$ & 4 \\
\hline $149757^{\ddagger}$ & $*$ & 6.3 & 23.6 & 140 & (16) & 21.13 & $-0.4(1)$ & $-1.6(1)$ & $-1.3(1)$ & $0.1(4)$ & $-2.1(1)$ & $-0.7(1)$ & $1,2,4$ \\
\hline & & & & & & 19.74 & $0.0(3)$ & $-0.9(1)$ & $-0.5(1)$ & & $-1.1(1)$ & $0.0(1)$ & 2 \\
\hline 149881 & & 31.4 & 36.2 & 2100 & & 20.57 & & $0.0(2)$ & $-0.3(3)$ & $-0.1(2)$ & -0.8 & $0.0(3)$ & 2,4 \\
\hline 154368 & $*$ & 350.0 & 3.2 & 366 & (199) & 21.62 & $-0.5(1)$ & $-0.6(3)$ & $-0.8(5)$ & $-0.6(3)$ & $-1.2(3)$ & $0.1(3)$ & 2 \\
\hline 157246 & $*$ & 334.6 & -11.5 & 348 & (123) & 20.73 & $-0.3(1)$ & & & & & & 1 \\
\hline 158926 & $*$ & 351.8 & -2.2 & 216 & $(52)$ & 19.23 & & & & & & $-0.1(3)$ & 4 \\
\hline 167756 & & 351.5 & -12.3 & 4000 & & 20.81 & & $-0.9(1)$ & & & & $-0.2(1)$ & 2,4 \\
\hline 212571 & $*$ & 66.0 & -44.7 & 338 & (107) & 20.56 & & & & $-0.3(3)$ & $-0.9(4)$ & $0.0(3)$ & 2,4 \\
\hline 215733 & & 85.2 & -36.4 & 2900 & & 20.76 & & $-0.2(3)$ & $-0.6(2)$ & & & $-0.3(2)$ & 4,6 \\
\hline $3 \mathrm{C} 273$ & $*$ & 290.0 & 64.4 & & & 20.10 & & & $-0.7(2)$ & $0.1(2)$ & & & 2 \\
\hline
\end{tabular}

References: (1) Meyer et al. (1998), (2) references given in Savage \& Sembach (1996a, their Table 3),

(3) Savage \& Sembach (1996b), (4) Roth \& Blades (1995), (5) Dupin \& Gry (1998), (6) Fitzpatrick \& Spitzer (1997),

$\dagger$ Dubner et al. (1998), ${ }^{\ddagger}$ cool (first row) and warm (second row) clouds.

singly ionized $\mathrm{Na}$ is more abundant in the ISM. Therefore these abundances cannot be compared directly to that of the other elements, except for the sight line to $\zeta$ Oph (HD 149757). For this star, a ratio of the ionized to neutral Na column density of 3.1 was derived by Morton (1975).

\subsection{Mean abundances}

The depletions of the individual elements in the ISM have been averaged over all sight lines and the results are displayed in Fig. 4, as well as the highest and lowest measured relative abundances. For most elements the abundances vary considerably for the different regions that have been analysed. For comparison, the $\lambda$ Bootis star abundance pattern (Paper I) is also shown in Fig. 4 (grey bars). The scatter of element abundances within the two samples is similar, except for $\mathrm{O}$, which seems to be distributed very homogeneously throughout the ISM. Another similarity between the $\lambda$ Bootis group and the ISM are the mean abundances of $\mathrm{C}$ and $\mathrm{O}$, which are slightly below solar. For the abundances of $\mathrm{Na}$, the ratio of ionized to neutral $\mathrm{Na}$ in the direction of $\zeta \mathrm{Oph}$ has been adopted for all 


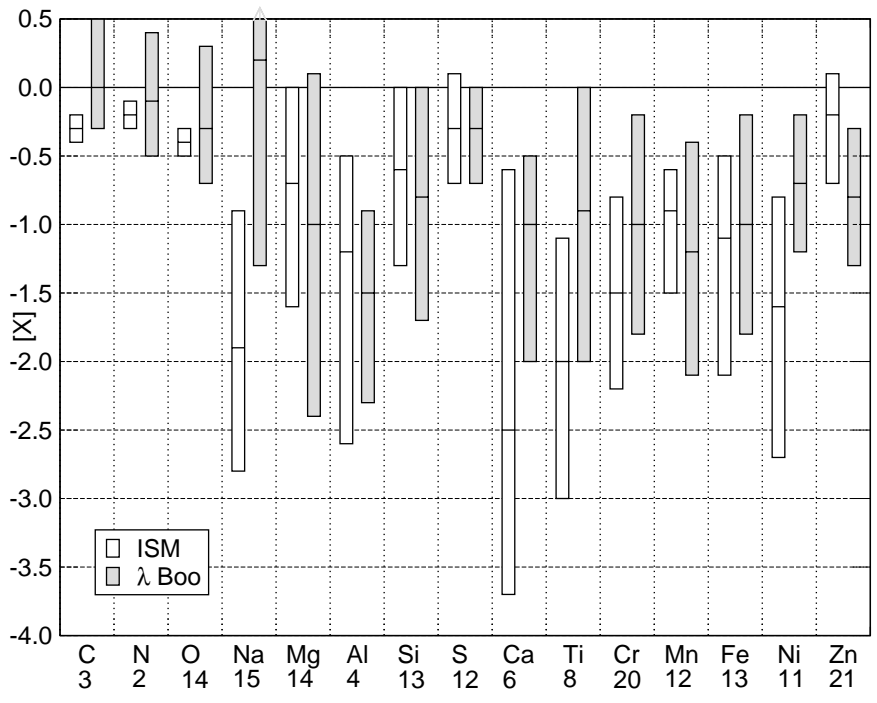

Fig. 4. Mean abundances for all ISM sight lines from Table 3, as well as highest and lowest abundances. The number of available abundances is given below the element name. The grey bars indicate the mean and ranges of $\lambda$ Bootis star abundances.

other sight lines, although the validity of this assumption is unsettled.

For S, the ISM abundance distribution is exactly the same as for the $\lambda$ Bootis stars. On the other hand, the mean abundance of $\mathrm{Zn}$ is significantly lower (by $0.6 \mathrm{dex}$ ) in $\lambda$ Bootis stars than in the ISM. Also, the mean abundances of $\mathrm{Mg}, \mathrm{Al}, \mathrm{Si}$ and $\mathrm{Mn}$ are slightly lower (but note that only four ISM measurements are available for $\mathrm{Al}$ ). Furthermore, for all of these elements except $\mathrm{Al}$ the lowest abundances are lower in the $\lambda$ Bootis stars than in the ISM by 0.4 to 0.8 dex.

This does not seem to fit into the accretion/diffusion theory, because the originally normal element abundance in the stellar atmosphere should converge to the abundance in the accreted (interstellar) matter. The ultimate atmospheric abundance depends mainly on the accretion rate and the mass of the convection zone. In this picture the ISM abundances set a lower limit for the $\lambda$ Bootis star abundances, which is exceeded by the observations. The situation is different for the other heavier elements, where the mean ISM abundances lie well below the mean $\lambda$ Bootis abundances, and also the lowest ISM abundances are lower than that of the $\lambda$ Bootis stars. The most controversial abundances are that of $\mathrm{Zn}$, whose mean abundance and distribution is similar to that of $\mathrm{S}$ in the ISM. The mean $[\mathrm{Zn} / \mathrm{Fe}]$ ratio is $+1.0 \pm 0.3 \mathrm{dex}$ in the ISM, whereas the mean ratio of the same elements for $\lambda$ Bootis stars is $+0.3 \pm 0.4$ dex. These values have been derived including only sight lines or stars for which the abundances of both elements are available. We obtain the same values when using only Fe I lines for the $\lambda$ Bootis stars, thus possible unconsidered Zn I NLTE effects seem to be negligible (see also Paper I, Sect. 4).

The significance of these findings could be reduced by the fact that interstellar depletions depend on cloud

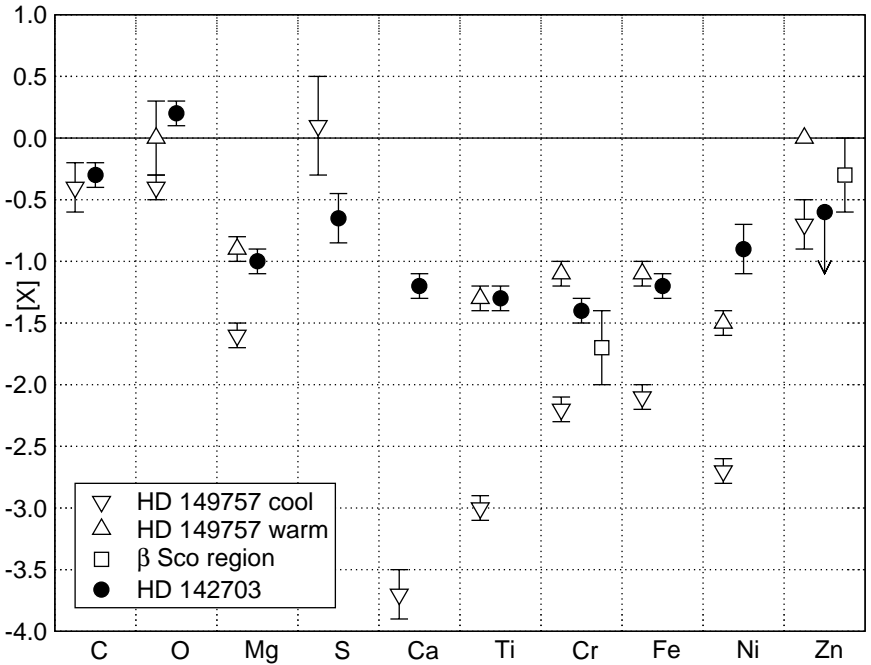

Fig. 5. Comparison of the interstellar abundances towards the regions of HD 149757 and $\beta$ Sco (representing the sight lines towards HD 141637, HD 143018, HD 144217 and HD 147165) with that of the $\lambda$ Bootis star HD 142703 .

conditions (Savage \& Sembach 1996a; Spitzer 1985), but we have averaged over a large range of densities (and temperatures). However, if we divide the ISM sample into clouds with "low" $(\log N(\mathrm{H})<20.5)$ and "high" $(\log N(\mathrm{H})>20.5)$ densities the average depletions of the deficient elements are larger in the denser (and cooler) clouds by only 0.3 dex, although the minimum abundances are on the average by 0.9 dex lower. Therefore, the problems discussed in the previous paragraph remain, in particular if we regard the circumstellar environment to be represented more closely by the less dense (and warmer) interstellar clouds.

\subsection{Individual sight lines}

We compared also the abundance patterns of individual $\lambda$ Bootis stars with that of nearby ISM sight lines. The extension of the interstellar cloud in the direction of HD 154368 is given by Snow et al. (1996) as $l=$ $346^{\circ} \ldots 25^{\circ}, b=-6^{\circ} \ldots+6^{\circ}, r=125 \pm 25$ pc. This region is located near the $\lambda$ Bootis star HD 170680. In analogy, sight lines having $\lambda$ Bootis stars with a $(l, b)$ distance of less than about $15^{\circ}$ in their vicinity have been marked with an asterisk in Table 3. This corresponds to linear distances of about ten to $60 \mathrm{pc}$, depending on the distances of the $\lambda$ Bootis stars from the Sun. The respective $\lambda$ Bootis stars are less distant than each of the ISM stars. Only few common element abundances are available. They are listed in Table 4, where upper rows and lower rows correspond to ISM sight lines and $\lambda$ Bootis stars, respectively. One region with more elements available for a comparison is shown in Fig. 5.

Of particular interest is the region in the direction of HD 93521, for which six elements can be compared with the stars HD 84123, HD 84948 and HD 101108. The ISM 
Table 4. Comparison of interstellar abundances (first rows) with that of $\lambda$ Bootis stars (second/third rows). The Orion region corresponds to the sight lines to the stars HD 35149, HD 36486, HD 36861 and HD 37128, and the $\lambda$ Sco region corresponds to HD 154368 and HD 158926.

\begin{tabular}{|c|c|c|c|c|c|c|c|c|c|c|c|}
\hline & $\mathrm{C} / \mathrm{C}_{\mathrm{NIR}}$ & $\mathrm{O} / \mathrm{O}_{\mathrm{NIR}}$ & $\mathrm{Na}$ & $\mathrm{Mg}$ & $\mathrm{Si}$ & $\mathrm{S}$ & $\mathrm{Ca}$ & $\mathrm{Ti}$ & $\mathrm{Cr}$ & $\mathrm{Mn}$ & $\mathrm{Fe}$ \\
\hline ISM (HD 22586) & & & & & $-0.6(2)$ & & $-2.4(2)$ & $-1.7(2)$ & & & $-1.1(2)$ \\
\hline HD 11413 & & & & & $-0.2(2)$ & & $-0.9(3)$ & $-1.4(2)$ & & & $-1.5(2)$ \\
\hline ISM (Orion region) & & $-0.4(1)$ & $-2.1(3)$ & & & & & & $-1.7(3)$ & & \\
\hline HD 31295 & & $-0.5(1) / 0.0(1)$ & $-0.5(2)$ & & & & & & $-0.8(2)$ & & \\
\hline ISM (HD 93521) & & & & $+0.0(4)$ & $-0.4(2)$ & $+0.0(1)$ & & $-1.1(5)$ & & $-0.7(3)$ & $-0.9(4)$ \\
\hline HD 84123 & & & & $-1.0(2)$ & $-1.0(2)$ & $-0.6(1)$ & & $-1.0(1)$ & & $-1.1(3)$ & $-1.2(2)$ \\
\hline HD $84948 \mathrm{~A}$ & & & & $-1.2(5)$ & $-0.8(4)$ & & & $-1.3(3)$ & & & $-1.2(3)$ \\
\hline HD $84948 \mathrm{~B}$ & & & & $-1.0(4)$ & $-0.6(5)$ & & & $-0.5(4)$ & & & $-1.0(2)$ \\
\hline HD 101108 & & & & $-0.3(2)$ & $-0.5(3)$ & & & $-0.2(2)$ & & $-0.4(2)$ & $-0.7(1)$ \\
\hline ISM (3C 273) & & & & & $-0.7(2)$ & & & & & $-0.7(1)$ & \\
\hline HD 105759 & & & & & & & & & & $-1.0(5)$ & \\
\hline HD 110411 & & & & & $<-0.3$ & & & & & & \\
\hline ISM (HD 157246) & & $-0.3(1)$ & & & & & & & & & \\
\hline HD 168740 & & $/ 0.0(1)$ & & & & & & & & & \\
\hline ISM ( $\lambda$ Sco region) & $-0.4(2)$ & $-0.5(1)$ & & $-0.6(3)$ & & & & $-2.5(3)$ & $-1.1(3)$ & & $-1.2(3)$ \\
\hline HD 170680 & $/ 0.0(1)$ & $/ 0.0(1)$ & & $-0.2(2)$ & & & & $-0.5(1)$ & $-0.4(3)$ & & $-0.4(1)$ \\
\hline ISM (HD 212571) & & & & & & $-0.3(3)$ & & & $-1.4(3)$ & & \\
\hline HD 204041 & & & & & & $+0.0(2)$ & & & $-0.8(2)$ & & \\
\hline
\end{tabular}

abundances of the lighter elements $\mathrm{Mg}, \mathrm{Si}$ and $\mathrm{S}$ are too high to explain the $\lambda$ Bootis abundances by accretion of this (or similar) material. On the other hand, the abundances of the heavy elements Ti, Mn and Fe are similar in this ISM region and the three $\lambda$ Bootis stars. The averaged $\mathrm{Cr}$ and $\mathrm{Zn}$ abundances of four adjacent sight lines (HD 141637, HD 143018, HD 144217 and HD $147165=$ " $\beta$ Sco region"), the abundance pattern of the most intensively studied $\zeta$ Oph sight line and that of the $\lambda$ Bootis star HD 142703 are displayed in Fig. 5. All ISM abundances except for $\mathrm{S}$ are lower than in the $\lambda$ Bootis star, when regarding the cool $\zeta$ Oph cloud. For the warm cloud, the $\mathrm{Cr}$ and $\mathrm{Zn}$ abundances are also discrepant. For the other regions we observe the same trends in the individual abundance patterns as for the mean values.

In conclusion, the accretion scenario could explain the spectroscopic features of a fraction of the $\lambda$ Bootis stars. For the other part, the observed ISM underabundances are not low enough, which suggests that either a different mechanism is operating or the circumstellar abundances differ significantly from that measured in the near interstellar medium. It might also be possible that the local abundance variations in the ISM are larger than assumed for the comparison. Finally, there could be additional uncertainties in the stellar abundances because of unaccounted-for physical processes in the atmospheres, like stratification due to diffusion, or inadequate treatment of convection.

\section{Comparison with stars surrounded by circumstellar matter}

The post- $A G B$ stars show evidence for being surrounded by circumstellar shells. A small subgroup with unusually low metal abundances, but nearly solar abundances

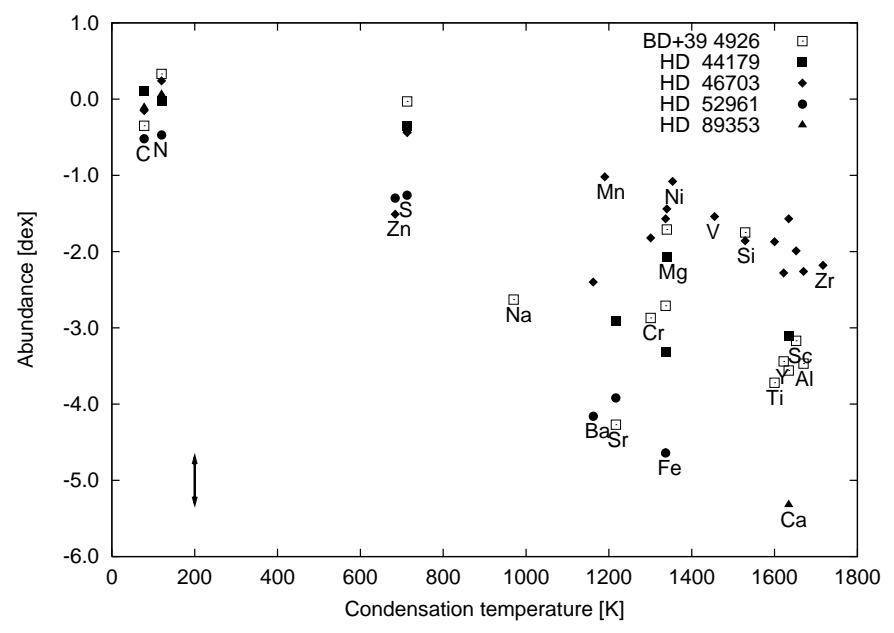

Fig. 6. Abundances of metal poor post-AGB stars versus condensation temperature. The arrow to the lower left represents the mean error of $s$.

of C, N, O and S has been identified (Bond 1991). It has been proposed that they have experienced accretion of the circumstellar gas depleted by dust fractionation (van Winckel et al. 1992), which is supported by an enhanced abundance of $\mathrm{Zn}$ relative to $\mathrm{Fe}$ in one of these stars. Figure 6 shows the correlation between the abundances of the five metal poor post-AGB stars (Kodaira 1973; Lambert et al. 1988; Luck \& Bond 1984; Bond \& Luck 1987; Waelkens et al. 1991; Waelkens et al. 1992; van Winckel et al. 1992) and the condensation temperatures $\left(T_{\mathrm{c}}\right)$ of the elements (taken from Fegley 1997). A similar but more shallow correlation (with one or two exceptional elements) can be seen in HD 106223 (Fig. 7; note that the vertical scale is different from Fig. 6) and six other $\lambda$ Bootis stars (HD 319, HD 31295, HD 75654, HD 142703, 


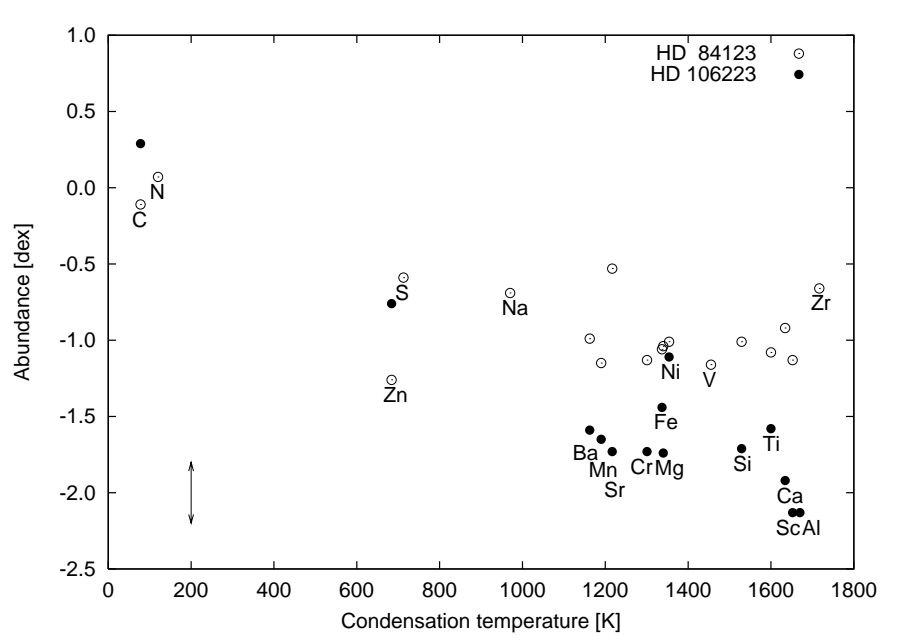

Fig. 7. Abundances of the $\lambda$ Bootis stars HD 84123 and HD 106223 versus condensation temperature. The arrow to the lower left represents the mean error of the abundances.

HD 204041 and HD 210111). Other $\lambda$ Bootis stars show nearly solar abundances of elements with low condensation temperature $(\mathrm{C}, \mathrm{N}$, in some cases also $\mathrm{S}$ and $\mathrm{Na}$ ) and more or less constant underabundances of the other elements like HD 84123 in Fig. 7. We conclude that chemical separation could be responsible for the abundances of some, but not all $\lambda$ Bootis stars, although the mechanism seems to be less efficient than in the post-AGB stars. For the comparison of the abundances of the two groups, it has to be taken into account that the original mean abundance in post-AGB stars is low $(\approx-0.7 \mathrm{dex}$, see Heiter 2000) because of their age (population II). Furthermore, their $\mathrm{C}, \mathrm{N}$ and $\mathrm{O}$ abundances have been enhanced during the AGB phase.

"Vega-like" stars exhibit IR flux excesses which indicate the presence of a dust envelope. Many members of this class have been found through searches of the IRAS catalog (Mannings \& Barlow 1998, and references therein). Three $\lambda$ Bootis stars are amongst them: $\lambda$ Boo, $\pi^{1}$ Ori and $\rho$ Vir. Table 5 summarizes the results of dedicated searches for IR excesses in $\lambda$ Bootis stars including data obtained with the ISO satellite. As the dust detection rate is very small, the existence of shells around most $\lambda$ Bootis stars, as required by the accretion/diffusion hypothesis, has to be questioned. Otherwise, the excess IR emission might be too faint to be detected with today's IR photometric devices. Holweger et al. (1999) investigated high resolution spectra of normal and "dusty" A stars and $\lambda$ Bootis stars (Table 5). They concluded that signatures of circumstellar gas are not as common as that of circumstellar dust in normal A stars and that either the two kinds of circumstellar matter rarely coexist around a star (they could appear at different evolutionary stages) or that both components cannot be detected at the same time.

Abundances are only available for seven "dusty" A type main sequence stars (Dunkin et al. 1997; Holweger et al. 1997), apart from Vega (Adelman \& Gulliver 1990;
Table 5. Dust and gas around $\lambda$ Bootis stars. The abbreviations for the dust references are as follows: S1986 - Sadakane \& Nishida (1986), C1992 - Cheng et al. (1992), K1994 - King (1994), F1999 - Fajardo-Acosta et al. (1999), P2000 - Paunzen (2000). The references for gas detection are: 1 - Holweger et al. (1999), 2 - Hauck et al. (1998), 3 - Hauck et al. (1995).

\begin{tabular}{lllll}
\hline \hline HD & Dust? & Ref. & Gas? & Ref. \\
\hline 319 & $?$ & - & no & 1 \\
11413 & probably & F1999 & yes & 1 \\
30422 & $?$ & - & no & 1 \\
31295 & yes & K1994, S1986 & no & 1,2 \\
109738 & no & K1994 & $?$ & - \\
110411 & yes & F1999, C1992 & no & 1,2 \\
111604 & $?$ & - & no & 2 \\
125162 & yes & P2000, K1994, & no & 2 \\
& & S1986 & & \\
142703 & probably & P2000 & no & 1 \\
154153 & no & K1994 & $?$ & - \\
183324 & $?$ & - & no & 1 \\
192640 & no/yes & P2000/F1999 & no & 3 \\
193256 & $?$ & - & yes & 1 \\
193281 & $?$ & - & no & 1 \\
$198160 / 1$ & $?$ & - & yes & 1 \\
204041 & probably & P2000 & no & 1 \\
210111 & $?$ & - & no & 1 \\
221756 & no & P2000 & no & 2 \\
\hline \hline
\end{tabular}

Ilijic et al. 1998). Two of them ( $\beta$ Pic and $\gamma$ Oph) additionally show evidence for circumstellar gas shells in their spectra. No abundances are available for any other spectroscopically classified "A-shell" stars. The abundances of Vega lie in the range of the $\lambda$ Bootis star abundances, except for $\mathrm{Al}, \mathrm{V}$ and $\mathrm{Zr}$, which are more abundant than in any of the $\lambda$ Bootis stars. This abundance pattern provides only weak evidence against a classification of Vega as a $\lambda$ Bootis star. But a recent analysis of IUE spectra (E. Solano, private communication), shows that Vega has to be excluded from the $\lambda$ Bootis group, because the $\mathrm{C} /(\mathrm{Al}, \mathrm{Si}, \mathrm{Ca})$ equivalent width ratios are by far smaller than the limiting criteria defined by Solano \& Paunzen (1998, 1999). However, Vega is the most metal deficient of the analysed Vega-like stars, with only one star (HD 169142) reaching similar underabundances for $\mathrm{Mg}$ and Si. All other element abundances correspond to that of normal stars (Paper I). These results show that presence of circumstellar matter around A-type main sequence stars is not necessarily related to abundance anomalies as observed in $\lambda$ Bootis stars.

\section{Conclusions}

The $\lambda$ Bootis star abundances were examined with regard to correlations to the stellar parameters of this group, in particular the effective temperature. It was found that for some elements (C, Na, Mg, Si, Ca, Sc, Cr, Fe, Sr) the abundances are weakly correlated with $T_{\text {eff }}, \log g$, the age, the pulsational period or $v \cdot \sin i$. The scatter of heavy element abundances in individual stars does not depend on 
Table 6. Summary of the search for signatures of accretion. $\sigma \ldots$ cool stars with small $(+)$ or large $(-) \sigma$ from Table 1 , hot stars with $[\mathrm{Ca}]=[\mathrm{Ti}](\mathrm{x}) ; I \ldots$ all abundances higher than in ISM $(+)$, some abundances lower than in ISM $(-)$; $C \ldots$ smooth $(+)$ or discontinuous $(-)$ relation of abundances and $T_{\mathrm{c}}$

\begin{tabular}{rlll|rrrr}
\hline \hline HD & $\sigma$ & $I$ & $C$ & HD & $\sigma$ & $I$ & $C$ \\
\hline 319 & + & & + & 125162 & - & & - \\
11413 & - & + & - & 142703 & + & - & + \\
15165 & - & & - & 168740 & + & & \\
31295 & $\times$ & + & + & 170680 & & + & \\
74873 & $\mathrm{x}$ & & - & 183324 & $\mathrm{x}$ & & - \\
75654 & & & + & 192640 & + & & - \\
84123 & + & - & - & 193256 & - & & - \\
$84948 \mathrm{~A} / \mathrm{B}$ & + & - & & $198160 / 1$ & - & & \\
101108 & + & + & - & 204041 & - & + & + \\
106223 & + & & + & 210111 & - & & + \\
107233 & + & & & 221756 & $\mathrm{x}$ & & \\
110411 & $\mathrm{x}$ & & - & & & & \\
\hline \hline
\end{tabular}

$T_{\text {eff }}$. These findings are inconclusive with regard to testing the accretion/diffusion theory. Because of the lack of calculations for more than three elements and different atmospheric parameters, the treatment of convection, the calculation of the radiative acceleration and the free parameters (mainly the accretion rate and the abundance spectrum in the accreted material) we consider the theoretical models to be rather simple and incomplete.

The chemical composition of the $\lambda$ Bootis stars has been compared to that of the interstellar medium (ISM). The mean abundances of some elements (Mg, Si, Mn, Zn) are slightly lower in the $\lambda$ Bootis stars than in the ISM (by 0.2 to $0.6 \mathrm{dex}$ ), and the lowest abundances found in $\lambda$ Bootis stars for these elements are lower than the lowest ISM abundances by 0.4 to 0.8 dex. Similar deviations have been found for only half of the single stars which can be compared to nearby sight lines. Within an accretion/diffusion scenario, the abundances of the accreted elements would be expected to be greater than in the ISM.

The $\lambda$ Bootis abundance pattern has also been compared to that of stars with circumstellar material (postAGB, Vega-like and A-shell stars). Similar relations of abundances with condensation temperatures suggest that the same physical processes lead to the chemical compositions of some $\lambda$ Bootis stars and the metal poor postAGB stars although theoretical calculations for the latter group do not exist. More observations are clearly needed to confirm this hypothesis. On the other hand, the lack of metal deficiency in dusty stars with atmospheric parameters similar to $\lambda$ Bootis stars questions the connection of circumstellar dust with the $\lambda$ Bootis phenomenon, although the comparison is based on a very small sample of Vega-like stars.

From the currently available abundance data we conclude that the stars HD 319, HD 31295 and HD 106223 could well have experienced accretion of circumstellar gas (see Table 6), which however has not been detected in their spectra. For the other stars, further examination and spectral data are required.

Acknowledgements. This research was carried out within the working group Asteroseismology-AMS, supported by the Fonds zur Förderung der wissenschaftlichen Forschung (project $S$ 7303-AST). We want to thank E. Solano for providing the information on the IUE spectra of Vega. Thanks go to the referee, K. A. Venn, whose comments have helped to greatly improve the paper. Use was made of the Simbad database, operated at CDS, Strasbourg, France.

\section{References}

Adelman, S. J., \& Gulliver, A. F. 1990, ApJ, 348, 712

Andrievsky, S. M. 1997, A\&A, 321, 838

Andrievsky, S. M., \& Paunzen, E. 2000, MNRAS, 313, 547

Bond, H. E. 1991, in Evolution of Stars: The Photospheric Abundance Connection, ed. G. Michaud, \& A. Tutukov (Netherlands: IAU), 341

Bond, H. E., \& Luck, R. E. 1987, ApJ, 312, 203

Charbonneau, P. 1991, ApJ, 372, L33

Charbonneau, P. 1993, ApJ, 405, 720

Charbonneau, P., \& Michaud, G. 1991, ApJ, 370, 693

Claret, A. 1995, A\&AS, 109, 441

Dubner, G. M., Green, A. J., Goss, W. M., Bock, D. C., \& Giacani, E. 1998, AJ, 116, 813

Dunkin, S. K., Barlow, M. J., \& Ryan, S. G. 1997, MNRAS, 286,604

Dupin, O., \& Gry, C. 1998, A\&A, 335, 661

Faraggiana, R., \& Bonifacio, P. 1999, A\&A, 349, 521

Fegley, B. 1997, in Encyclopedia of Planetary Sciences, ed. J. H. Shirley, \& R. W. Fairbridge (London: Chapman \& Hall), 169

Fitzpatrick, E. L., \& Spitzer, L. 1997, ApJ, 475, 623

Grevesse, N., Noels, A., \& Sauval, A. J. 1996, in Cosmic Abundances, ASP Conf. Ser., 99, 117

Handler, G. 1995, Inf. Bull. Variable Stars, 4216

Hauck, B., \& Mermilliod, M. 1990, A\&AS, 86, 107

Heiter, U. 2000, Ph.D. Thesis, Univ. of Vienna, Austria, available as postscript file at

http://ams.astro.univie.ac.at/ ^heiter/thesis/ thesis.ps.gz

Heiter, U. 2002, A\&A, 381, 959

Holweger, H., Hempel, M., \& Kamp, I. 1999, A\&A, 350, 603

Holweger, H., Hempel, M., van Thiel, T., \& Kaufer, A. 1997, A\&A, 320, L49

Ilijic, S., Rosandic, M., Dominis, D., Planinic, M., \& Pavlovski, K. 1998, Contrib. Astron. Obs. Skalnaté Pleso, 27, 467

Kodaira, K. 1973, A\&A, 22, 273

Lambert, D. L., Hinkle, K. H., \& Luck, R. E. 1988, ApJ, 333, 917

Luck, R. E., \& Bond, H. E. 1984, ApJ, 279, 729

Mannings, V., \& Barlow, M. J. 1998, ApJ, 497, 330

Martinez, P., Koen, C., Handler, G., \& Paunzen, E. 1998, MNRAS, 301, 1099

Meyer, D. M., Jura, M., \& Cardelli, J. A. 1998, ApJ, 493, 222

Michaud, G., \& Charland, Y. 1986, ApJ, 311, 326

Morel, P. 1997, A\&AS, 124, 579

Morton, D. C. 1975, ApJ, 197, 85 
Paunzen, E. 2000, Ph.D. Thesis, Univ. of Vienna, Austria, available on request from the author

Paunzen, E., Kuschnig, R., Handler, G., Gelbmann, M., \& Weiss, W. W. 1997, A\&AS, 124, 23

Paunzen, E., Weiss, W. W., Kuschnig, R., et al. 1998, A\&A, 335,533

Perryman, M. A. C., \& ESA 1997, The HIPPARCOS and TYCHO catalogues. Astrometric and photometric star catalogues derived from the ESA HIPPARCOS Space Astrometry Mission (ESA SP Ser. 1200)

Roth, K. C., \& Blades, J. C. 1995, ApJ, 445, L95

Savage, B. D., \& Sembach, K. R. 1996a, RA\&A, 34, 279

Savage, B. D., \& Sembach, K. R. 1996b, ApJ, 470, 893

Schmidt-Kaler, T. 1982, in Landolt-Börnstein New Series, vol. VI 2b, ed. K. Schaifers, \& H. H. Voigt (Heidelberg: Springer)
Snow, T. P., Black, J. H., Dishoeck, E. F. V., et al. 1996, ApJ, 465,245

Solano, E., \& Paunzen, E. 1998, A\&A, 331, 633

Solano, E., \& Paunzen, E. 1999, A\&A, 348, 825

Spitzer, L. 1985, ApJl, 290, L21

Turcotte, S., \& Charbonneau, P. 1993, ApJ, 413, 376

van Winckel, H., Mathias, J., \& Waelkens, C. 1992, Nature, 356,500

Venn, K. A., \& Lambert, D. L. 1990, ApJ, 363, 234

Waelkens, C., van Winckel, H., Bogaert, E., \& Trams, N. R. 1991, A\&A, 251, 495

Waelkens, C., van Winckel, H., Trams, N. R., \& Waters, L. B. F. M. 1992, A\&A, 256, L15

Welty, D. E., Hobbs, L. M., \& Kulkarni, V. P. 1994, ApJ, 436, 152 